\title{
Review on Diagnosis of COVID-19 from Chest CT Images Using Artificial Intelligence
}

\author{
Ilker Ozsahin $\mathbb{D}^{1,2}$ Boran Sekeroglu $\mathbb{D}^{1,2,3}$ Musa Sani Musa $\mathbb{D}^{1},{ }^{1}$ Mubarak Taiwo Mustapha, ${ }^{1,2}$ \\ and Dilber Uzun Ozsahin $\mathbb{i D}^{1,2}$ \\ ${ }^{1}$ Department of Biomedical Engineering, Near East University, Nicosia / TRNC, Mersin-10, 99138, Turkey \\ ${ }^{2}$ DESAM Institute, Near East University, Nicosia / TRNC, Mersin-10, 99138, Turkey \\ ${ }^{3}$ Department of Artificial Intelligence Engineering, Near East University, Nicosia / TRNC, Mersin-10, 99138, Turkey
}

Correspondence should be addressed to Ilker Ozsahin; ilker.ozsahin@neu.edu.tr

Received 26 June 2020; Revised 28 August 2020; Accepted 16 September 2020; Published 26 September 2020

Academic Editor: Lin Lu

Copyright ( 2020 Ilker Ozsahin et al. This is an open access article distributed under the Creative Commons Attribution License, which permits unrestricted use, distribution, and reproduction in any medium, provided the original work is properly cited.

\begin{abstract}
The COVID-19 diagnostic approach is mainly divided into two broad categories, a laboratory-based and chest radiography approach. The last few months have witnessed a rapid increase in the number of studies use artificial intelligence (AI) techniques to diagnose COVID-19 with chest computed tomography (CT). In this study, we review the diagnosis of COVID-19 by using chest CT toward AI. We searched ArXiv, MedRxiv, and Google Scholar using the terms "deep learning", "neural networks", "COVID-19", and "chest CT". At the time of writing (August 24, 2020), there have been nearly 100 studies and 30 studies among them were selected for this review. We categorized the studies based on the classification tasks: COVID19/normal, COVID-19/non-COVID-19, COVID-19/non-COVID-19 pneumonia, and severity. The sensitivity, specificity, precision, accuracy, area under the curve, and F1 score results were reported as high as $100 \%, 100 \%, 99.62,99.87 \%, 100 \%$, and $99.5 \%$, respectively. However, the presented results should be carefully compared due to the different degrees of difficulty of different classification tasks.
\end{abstract}

\section{Introduction}

Coronaviruses have been around for many decades, and it has affected many animals/mammal species and human being. By March 11, 2020, the World Health Organization (WHO) [1] declared the new coronavirus called the COVID-19, a pandemic, and it has brought the entire globe into a compulsory lockdown. Coronavirus is a family of RNA viruses that is capable of causing significant viral pathogens in humans and animals. Corona is medium-sized viruses with the largest viral RNA genome known. Coronavirus infects both birds and mammals, but the bat is host to the largest number of the viral genotype of coronavirus. So, the bat is the host and does not get infected. It can, however, spread the virus to a human. As of $24^{\text {th }}$ of August 2020, there have been more than 23 million confirmed cases of coronavirus worldwide, with about 800,000 of such cases resulting in the death of the infected patient. This is spread around 216 countries, areas, or territories. However, around five million infected patients have recovered worldwide [2]. The USA, Brazil, India, and Russia are the top four countries with the highest number of cases. Around 90 million tests have conducted in China, followed by the USA, Russia, and India, with 72 million, 33 million, and 32 million tests, respectively [2].

Testing COVID-19 involves analyzing samples that indicate the present or past presence of severe acute respiratory syndrome-associated coronavirus 2 (SARS-CoV-2). The test is done to detect either the presence of the virus or of antibodies produced in response to infection. COVID-19 diagnostic approach is mainly divided into two broad categories, a laboratory-based approach, which includes point of care-testing, nucleic acid testing, antigens tests, and serology (antibody) tests. The other approach is using medical 
imaging diagnostic tools such as X-ray and computed tomography (CT) [3].

The laboratory-based tests are performed on samples obtained via nasopharyngeal swab, throat swabs, sputum, and deep airway material [4]. The most common diagnostic approach is the nasopharyngeal swab, which involves exposing a swab to paper strips containing artificial antibodies designed to bind to coronavirus antigens. Antigens bind to the strips and give a visual readout [4]. The process is pretty fast and is employed at the point of care. The nucleic acid test has low sensitivity between $60-71 \%$ [4]. On the other hand, Fang et al. [5] showed that radiologic methods could provide higher sensitivity than that of lab tests.

The use of medical imaging tools is the second approach of COVID-19 virus detection. These tools are playing an important role in the management of patients that are confirmed or suspected to be infected with the virus. It is worthy of note that without clinical suspicion, findings from X-ray, or CT images are nonspecific as many other diseases could have a similar pattern [6].

Thoracic CT scan is the imaging modality of choice that plays a vital role in the management of COVID-19. Thoracic CT has a high sensitivity for diagnosis of COVID-19 which makes it a primary tool for COVID-19 detection [5]. CT scan involves transmitting X-rays through the patient's chest, which are then detected by radiation detectors and reconstructed into high-resolution medical images. There are certain patterns to look out for in a chest CT scans which present themselves in different characteristic manifestations. The potential findings with 100\% confidence for COVID-19 in thoracic CT images are ground - glass opacity $(\mathrm{GGO}) \pm$ crazy - paving and consolidation, air bronchograms, reverse halo, and perilobular pattern [6].

The abovementioned findings are reports presented by a radiologist who specializes in interpreting medical images. Interpretation of these findings by expert radiologists does not have a very high sensitivity [4]. Artificial intelligence (AI) has been employed as it plays a key role in every aspect of COVID-19 crisis management. AI has proven to be useful in medical applications since its inception, and it became widely accepted due to its high prediction and accuracy rates. In the diagnosis stage of COVID-19, AI can be used to recognize patterns on medical images taken by CT. Other applications of AI include, but not limited to, virus detection, diagnosis and prediction, prevention, response, recovery, and to accelerate research [7]. AI can be used to segment regions of interest and capture fine structures in chest CT images, self-learned features can easily be extracted for diagnosis and other applications as well. A recent study showed that AI accurately detected COVID-19 and was also able to differentiate it from other lung diseases and communityacquired pneumonia [8]. In this study, we review the diagnosis of COVID-19 by using chest CT toward AI.

\section{Materials and Methods}

We searched ArXiv, MedRxiv, and Google Scholar for AI for COVID-19 diagnosis with chest CT. At the time of writing (August 24, 2020), there have been nearly 100 studies and only 17 of them were peer-reviewed papers. In total, 30 studies (17 peer-reviewed and 13 non-peer-reviewed papers) were selected for this review. We noticed that very different classification terms are reported by the authors such as "normal", "healthy", "other", "COVID-19", "non-COVID-19", "without COVID-19", "community-acquired pneumonia (CAP)", "other pneumonia", "bacterial pneumonia", "SARS”, "lung cancer", "type A influenza (influ-A)", and "severity". Therefore, we categorized the studies into four main tasks as follows: COVID-19/normal, COVID-19/non-COVID-19, COVID-19/non-COVID-19 pneumonia, and COVID-19 severity classification. COVID-19 group consists of COVID-19 patients. The normal group includes only healthy subjects. Non-COVID-19 group includes either one of the cases which is not COVID-19 or a combination of all other cases. The non-COVID-19 pneumonia group includes other types of pneumonia, which is not caused by COVID-19, such as viral or bacterial pneumonia, as well as influenza $A$ and SARS. Lastly, COVID-19 severity classification aims at classifying the COVID-19 cases as severe or nonsevere.

Since the rapid studies on the detection of COVID-19 in CT scans continue, the researchers who take into account the peer-review period in the journals share the results they obtained in their studies with other researchers and scientists as preprints in different publication environments. Machine learning is used to make decisions on tasks that people have difficulty making decisions or problems that require more stable decisions using both numerical and image-based data. A deep convolutional neural network (CNN) is the most widely used among machine learning methods. It is one of the first preferred neural networks, especially in imagebased problems, since it contains both feature extraction and classification stages and produces very effective results. In image-based COVID-19 researches, the CNN model or different models produced from CNN are widely encountered. In the researches, a generally hold-out method and a few $k$-fold cross-validation were used during the training phase. In the hold-out method, while training is done by dividing the data into two parts as test and train, in $k$-fold cross-validation, the data is divided into $k$-folds, and the folds are trained $k$-times by shifting the testing fold in each training so that each fold is used in the test phase. It is used as a better method for model evaluation.

\section{Results}

3.1. COVID-19/Normal Classification Studies. Alom et al. [9] implemented two deep learning models for COVID-19 detection and segmentation. Inception Recurrent Residual Neural Network (IRRCNN), which is based on transfer learning, was used for the COVID-19 detection task, and the NABLA-N model was for the segmentation task. They considered different datasets to detect COVID-19 on CT images, by using an additional chest $\mathrm{X}$-ray dataset. The publicly available dataset was considered for the segmentation procedure of CT images, and the dataset that consists of 425 CT image samples, with 178 pneumonia, and 247 normal images were considered for the COVID-19 detection purpose. All images were resized to the dimensions of $192 \times$ 
192 pixels, and 375 of total images were used for training and validation with a data augmentation procedure. The training was performed using Adam optimizer with a learning rate of $1 \times 10^{-3}$ and a batch size of 16 . The COVID-19 detection and segmentation accuracy were achieved by $98.78 \%$ and $99.56 \%$, respectively.

$\mathrm{Hu}$ et al. [10] constructed an AI model on ShuffleNet V2 [11], which provides fast and accurate training in transfer learning applications. The considered CT dataset consists of 521 COVID-19 infected images, 397 healthy images, 76 bacterial pneumonia images, and 48 SARS images. The data augmentation procedure as flip, rotation, translation, brightness adjustment, and flip+brightness adjustment was applied in this study to increase the number of training images. The first experiment was performed on the classification of COVID19 images from normal healthy images. The average sensitivity, specificity, and area under the curve (AUC) score were obtained as $90.52 \%, 91.58 \%$, and 0.9689 , respectively.

Gozes et al. [12] proposed a comprehensive system to detect COVID-19 from normal cases. The proposed system included lung segmentation, COVID-19 detection in CT slices, and marking case as COVID-19 using a predetermined threshold based on the counted COVID-19 positive slices. Several datasets were considered in training and testing phases, and pretrained network ResNet50 was used for the detection of COVID-19. The sensitivity, specificity, and the AUC score were achieved as 94\%, 98\%, and 0.9940 , respectively.

In another study for differentiation of COVID-19 from normal cases, Kassani et al. [13] used several pretrained networks such as MobileNet [14], DenseNet [15], Xception [16], InceptionV3 [17], InceptionResNetV2 [18], and ResNet [19] to extract the features of images within the publicly available dataset. Then, extracted features were trained using six machine learning algorithms, namely, decision tree, random forest, XGBoost, AdaBoost, Bagging, and LightGBM. Kassani et al. [13] concluded that the Bagging classifier obtained the optimal results with a maximum of $99.00 \% \pm 0.09$ accuracy on features extracted by pretrained network DesnseNet121.

Jaiswal et al. [20] implemented a pretrained network DenseNet201-based deep model on classifying 2,492 CTscans (1,262 positive for COVID-19, and the rest 1,230 are negative) as positive or negative. They compared their results with VGG16, ResNet152V2, and Inception-ResNetV2. They concluded that their model outperformed other considered models and achieved an overall accuracy of $96.25 \%$. Table 1 summarizes the studies on COVID-19 vs. normal cases.

3.2. COVID-19/Non-COVID-19 Classification Studies. Jin et al. [30] considered 496 COVID-19 positive and 260 negative images collected in Wuhan Union Hospital, Western Campus of Wuhan Union Hospital, and Jianghan Mobile Cabin Hospital in Wuhan. Besides, they used two publicly available international databases, LIDC-IDRI [28] and ILDHUG [31] (1012 and 113 subjects, respectively) as negative cases to develop the system. A $2 \mathrm{D}$ convolutional neural network was used for the segmentation of CT slices, and then, a model was trained for positive and negative cases. Jin et al. reported that the proposed system achieved the AUC score of 0.9791 , sensitivity of $94.06 \%$, and specificity of 95.47\% for the external text cohort.

Singh et al. [32] proposed a multiobjective differential evolution- (MODE-) based convolutional neural networks to detect COVID-19 in chest CT images. It was concluded that the proposed method outperformed the CNN, ANFIS, and ANN models in all considered metrics between $1.6827 \%$ and $2.0928 \%$.

Amyar et al. [33] developed another model architecture that included image segmentation, reconstruction, and classification tasks, which was based on the encoder and convolutional layer. The experiments were performed on three datasets that included 1044 CT images, and the obtained results showed that the proposed architecture achieved the highest results in their experiment, with $0.93 \%$ of the AUC score.

Ahuja et al. [34] used data augmentation and pretrained networks to classify COVID-19 images. Data augmentation was performed using stationary wavelets, and the random rotation, translation, and shear operations were applied to the CT scan images. ResNet18, ResNet50, ResNet101, and SqueezeNet were implemented for the classification task, and Ahuja et al. concluded that ResNet18 outperformed other models by obtaining a 0.9965 AUC score.

Liu et al. [35] proposed another deep neural network model, namely, lesion-attention deep neural networks, where the backbone of the model used the weights of pretrained networks such as VGG16, ResNet18, and ResNet50. The proposed model was capable of classifying COVID-19 images, which was the main aim of the study, with 0.94 of the AUC score using VGG16 as the backbone model. Besides this, the model was able to make a multilabel prediction on the five lesions.

Instead of deep learning approaches, Barstugan et al. [36] considered machine learning algorithms to classify 150 COVID-19 and non-COVID-19 images. Several feature extraction methods such as grey-level size zone matrix (GLSZM) and discrete wavelet transform (DWT) were considered in the feature extraction process, and the extracted features were classified using a support vector machine. $K$ -fold cross-validations were performed in the experiments with 2, 5, and 10 folds. Barstugan et al. concluded that 99.68\% of accuracy was achieved by SVM using the GLSZM feature extraction method.

Wang et al. [37] conducted another study on differentiating COVID-19 from non-COVID-19 CT scans. In their proposed network, UNet was first trained for lung region segmentation, and then, they used a pretrained UNet to test CT volumes to obtain all lung masks. They concatenated CT volumes with corresponding lung masks and sent them to the proposed DeCoVNet for the training. Wang et al. concluded that the proposed network achieved a 0.959 ROC AUC score.

Chen et al. [38] performed a study on collected 46,096 images from 106 patients (Renmin Hospital of Wuhan University-Wuhan, Hubei province, China). The proposed system was based on segmenting CT scans using UNet++ and predicting the COVID-19 lesions. The prediction was performed by dividing an image into four segments and 
TABle 1: COVID-19/normal classification results. Class.: classification; bac. pneu.: bacterial pneumonia; Sens.: sensitivity; Spec.: specificity; Prec.: precision; Acc.: accuracy; AUC: area under the curve; Ref.: reference.

\begin{tabular}{|c|c|c|c|c|c|c|c|c|c|c|}
\hline Class. & Subjects & Dataset & Method & $\begin{array}{c}\text { Sens. }(\%) \\
\text { or recall }\end{array}$ & $\begin{array}{c}\text { Spec. } \\
(\%)\end{array}$ & $\begin{array}{c}\text { Prec. } \\
(\%)\end{array}$ & $\begin{array}{l}\text { Acc. } \\
(\%)\end{array}$ & $\begin{array}{c}\text { AUC } \\
(\%)\end{array}$ & $\begin{array}{l}\text { F1- } \\
\text { score }\end{array}$ & Ref. \\
\hline $\begin{array}{l}\text { COVID- } \\
\text { 19/normal }\end{array}$ & $\begin{array}{l}178 \text { pneumonia } \\
247 \text { normal }\end{array}$ & $\begin{array}{c}\text { Private }+ \\
{[21-23]}\end{array}$ & $\begin{array}{c}\text { DL } \\
\text { IRRCNN }\end{array}$ & N/A & N/A & N/A & 98.78 & N/A & 98.85 & $\begin{array}{c}\text { Alom et al. } \\
\text { [9] } \\
\text { Preprint }\end{array}$ \\
\hline $\begin{array}{l}\text { COVID- } \\
\text { 19/normal }\end{array}$ & $\begin{array}{l}521 \text { COVID-19 } \\
397 \text { normal } \\
76 \text { bac. pneu. } \\
48 \text { SARS }\end{array}$ & [24-26] & $\begin{array}{c}\text { DL } \\
\text { ShuffleNet } \\
\text { V2 }\end{array}$ & 90.52 & 91.58 & N/A & 91.21 & 96.89 & N/A & $\begin{array}{l}\text { Hu et al. } \\
\text { [10] } \\
\text { Preprint }\end{array}$ \\
\hline $\begin{array}{l}\text { COVID- } \\
\text { 19/normal }\end{array}$ & $\begin{array}{c}106 \text { COVID-19 } \\
100 \text { normal }\end{array}$ & $\begin{array}{c}\text { Private }+ \\
{[27,28]}\end{array}$ & $\begin{array}{c}\text { DL } \\
\text { ResNet50 }\end{array}$ & 98.2 & 92.2 & N/A & N/A & 99.6 & N/A & $\begin{array}{c}\text { Gozes et al. } \\
\text { [12] } \\
\text { Preprint }\end{array}$ \\
\hline $\begin{array}{l}\text { COVID- } \\
\text { 19/normal }\end{array}$ & $\begin{array}{c}\text { COVID-19: } \\
\text { X-ray:117; CT:20 } \\
\text { normal: X-ray:117; } \\
\text { CT:20 }\end{array}$ & $\begin{array}{l}{[21,22,} \\
29]\end{array}$ & $\begin{array}{c}\text { DenseNet121 } \\
+ \\
\text { Bagging }\end{array}$ & 99.00 & N/A & 99.00 & 99.00 & N/A & 99.00 & $\begin{array}{c}\text { Kassani et al. } \\
\text { [13] } \\
\text { Preprint }\end{array}$ \\
\hline $\begin{array}{l}\text { COVID- } \\
\text { 19/normal }\end{array}$ & $\begin{array}{c}\text { 1,262 COVID-19 } \\
\text { 1,230 normal }\end{array}$ & {$[23]$} & DenseNet201 & 96.29 & 96.21 & 96.29 & 96.25 & 97.0 & 96.29 & $\begin{array}{c}\text { Jaiswal et al. } \\
\text { [20] } \\
\text { Peer-reviewed }\end{array}$ \\
\hline
\end{tabular}

counting the consecutive images. If three consecutive images were classified as containing lesions, the case was classified as positive for COVID-19. The proposed system was evaluated using five different metrics, and it achieved $92.59 \%$ and $98.85 \%$ of accuracy in prospective and retrospective testing, respectively.

Jin et al. [39] considered the segmentation and pretrained models to classify COVID-19, healthy images, and inflammatory and neoplastic pulmonary diseases. Initially, preprocessing was applied to CT scan images to standardize images that were collected from five hospitals in China. Several segmentation models such as V-Net and 3D U-Net++ were considered, and segmented images were trained using pretrained network ResNet50 [19], Inception networks [17], DPN-92 [40], and Attention ResNet-50 [41]. Jin et al. concluded that the ResNet50 achieved the highest classification rates by 0.9910 of AUC score, $97.40 \%$ of sensitivity, and $92.22 \%$ of specificity with the images segmented by $3 \mathrm{D}$ U-Net++ segmentation model.

Pathak et al. [42] proposed a system for the detection of COVID-19 in CT scans that considered a preproposed transfer learning. The system used the ResNet50 to extract the features from CT images, and a 2D convolutional neural network was considered for the classification. The proposed system was tested on 413 COVID-19 and 439 non-COVID19 images with 10-fold cross-validation, and it achieved $93.01 \%$ of accuracy.

Polsinelli et al. [43] proposed a light architecture by modifying the CNN. The proposed model was tested on two different datasets, and several experiments with different combinations were performed. The proposed CNN achieved $83.00 \%$ of accuracy and 0.8333 of F1 score.

Han et al. [44] proposed a patient-level attention-based deep 3D multiple instance learning (AD3D-MIL) that learns Bernoulli distributions of the labels obtained by a pooling approach. They used a total of 460 chest CT examples, 230
CT examples from 79 COVID-19 confirmed patients, 100 CT examples from 100 patients with pneumonia, and 130 CT examples from 130 people without pneumonia. Their proposed model achieved an accuracy, AUC, and the Cohen kappa score of $97.9 \%, 99.0 \%$, and $95.7 \%$, respectively, in the classification of COVID-19 and non-COVID-19.

Harmon et al. [45] considered 2724 CT scans from 2617 patients in their study. Lung regions were segmented by using $3 \mathrm{~d}$ anisotropic hybrid network architecture (AH-Net), and the classification of segmented 3D lung regions was performed by using pretrained model DenseNet121. The proposed algorithm achieved an accuracy, specificity, and AUC score of $0.908,0.930$, and 0.949 , respectively. Table 2 shows the summary of the COVID-19/non-COVID-19 classification results.

3.3. COVID-19/Non-COVID-19 Pneumonia Classification Studies. $\mathrm{Xu}$ et al. [52] proposed a method that consisted of preprocessing, CT image segmentation using ResNet18, and the classification of CT scans performed by adding location-attention that provides the relative location information of the patch on the pulmonary image. The proposed method tested on the considered 618 CT samples (219 with COVID-19, 224 CT images with influenza-A viral, and 175 CT images for healthy people), and Xu et al. concluded that the overall accuracy rate of the proposed method was $86.7 \%$.

Wang et al. [53] proposed another deep learning method to distinguish COVID-19 and other pneumonia types. The segmentation, suppression of irrelevant area, and COVID19 analysis were the processes of the proposed method. DenseNet121-FPN [15] was implemented for lung segmentation, and COVID19Net that had a DenseNet-like structure was proposed for classification purposes. Two validation sets were considered, and the authors reported 0.87 and 0.88 ROC AUC scores for these validation sets. 


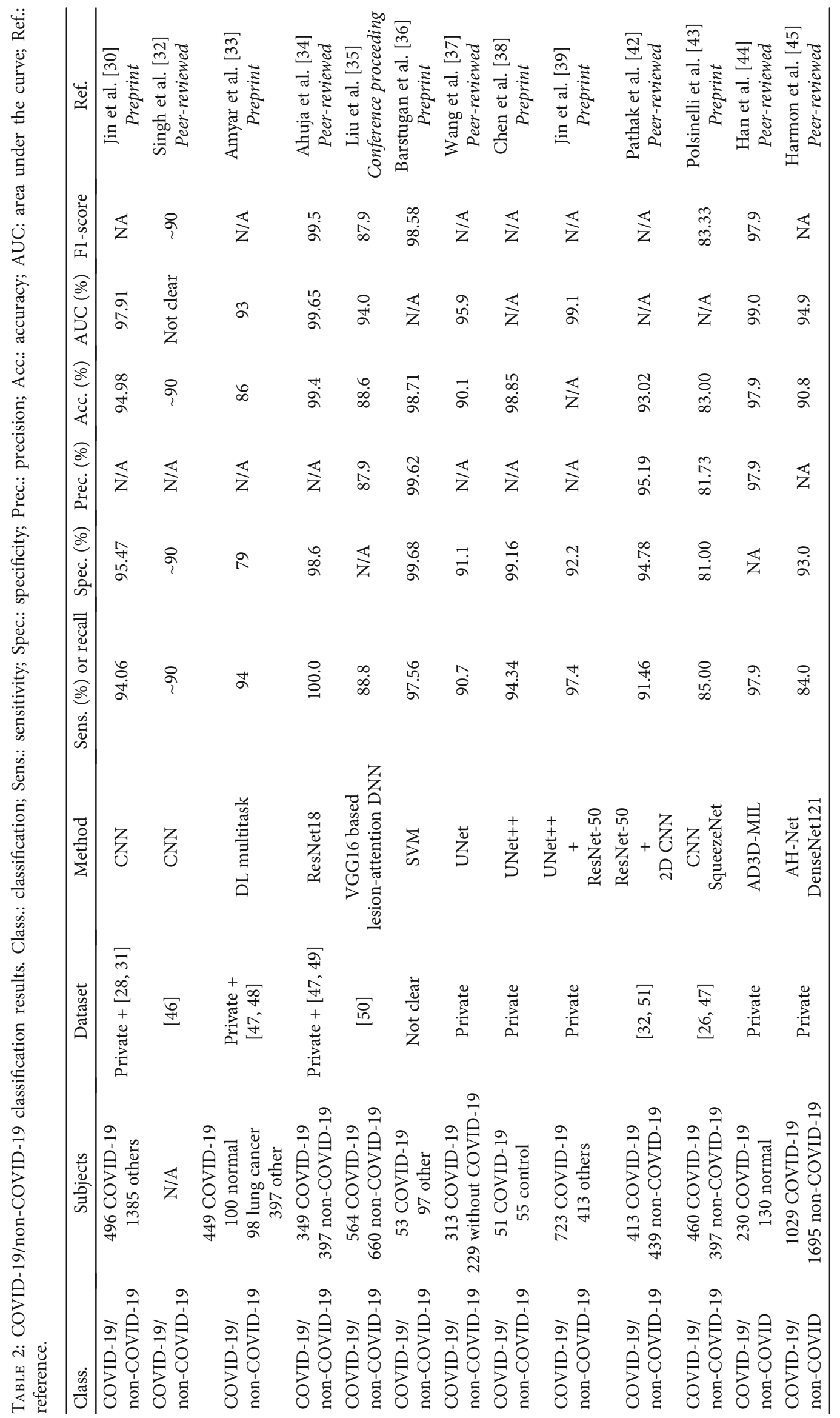


In addition to classify COVID-19 and normal cases, $\mathrm{Hu}$ et al. [10] performed another experiment to differentiate COVID-19 cases from other cases as bacterial pneumonia and SARS. The average sensitivity, specificity, and the AUC score were obtained as $0.8571,84.88 \%$, and $92.22 \%$, respectively.

Bai et al. [54] implemented the deep learning architecture EfficientNet B4 [55] to classify COVID-19 and pneumonia slices of CT scans. The diagnosis of the six radiologists on the corresponding patients were used to evaluate the efficiency of the results obtained by an AI model. The AI model achieved $96 \%$ of accuracy, while the average accuracy of the diagnosis of radiologists was obtained at $85 \%$.

Kang et al. [56] proposed a pipeline and multiview representation learning technique for COVID-19 classification using different types of features extracted from CT images. They used 2522 CT images (1495 are from COVID-19 patients, and 1027 are from communityacquired pneumonia) for the classification purpose. The comparison was performed using the benchmark machine learning models, namely, support vector machine, logistic regression, Gaussian-naive-Bayes classifier, $K$-nearestneighbors, and neural networks. The proposed method outperformed the considered ML models with 95.5\%, $96.6 \%$, and $93.2 \%$ in terms of accuracy, sensitivity, and specificity, respectively.

Another study was performed by Shi et al. [57] to classify COVID-19 and pneumonia. They considered 1658 and 1027 confirmed COVID-19 and CAP cases. Shi et al. proposed a model that is based on random forest and automatically extracted a series of features as volume, infected lesion number, histogram distribution, and surface area from CT images. The proposed method and considered machine learning models (logistic regression, support vector machine, and neural network) were then trained by the selected features with 5-fold cross-validation. The authors reported that the proposed method outperformed other models and produced the optimal AUC score (0.942).

Ying et al. [58] designed a network named as DRE-Net, which is based on the modifications on pretrained ResNet50. The CT scans of 88 COVID-19 confirmed patients, 101 patients infected with bacteria pneumonia, and 86 healthy persons. The designed network was compared by the pretrained models, ResNet, DenseNet, and VGG16. The presented results showed that the designed network outperformed other models by achieving 0.92 and 0.95 of AUC scores for the image and human levels.

In addition to COVID-19/non-COVID-19 classification, Han et al. [44] performed experiments to classify COVID-19, common pneumonia, and no pneumonia cases as three classes classification. Their proposed AD3D-MIL model achieved an accuracy, AUC, and the Cohen kappa score of $94.3 \%, 98.8 \%$, and $91.1 \%$, respectively.

Ko et al. [59] proposed a model, a fast-track COVID-19 classification network (FCONet) that used VGG16, ResNet50, InceptionV3, and Xception as a backbone to classify images as COVID-19, other pneumonia, or nonpneumonia. They considered 1194 COVID-19, 264 low-quality COVID19 (only for testing), and 2239 pneumonia, normal, and other disease CT scans in their study. All images were converted into grayscale image format with dimensions of $256 \times 256$. They used rotation and zoom data augmentation procedures to maximize the number of training samples. It was concluded that FCONet based on ResNet-50 outperformed other pretrained models and achieved $96.97 \%$ of accuracy in the external validation data set of COVID-19 pneumonia images.

Li et al. [8] proposed a COVNet that used ResNet50 as a backbone to differentiate COVID-19, nonpneumonia, and community-acquired pneumonia. In their study, 4352 chest CT scans from 3322 patients were considered. A maxpooling operation was applied to the features obtained from COVNet using the slices of the CT series, and the resultant feature map was fed to a fully connected layer. This led to generate a probability score for each considered class. It was concluded that the proposed model achieved a sensitivity, specificity, and ROC AUC scores of $90 \%, 96 \%$, and 0.96, respectively, for the COVID-19 class.

$\mathrm{Ni}$ et al. [60] considered a total of 19,291 CT scans from 14,435 individuals for their proposed model to detect COVID-19 in CT scans. Their proposed model included the combination of Multi-View Point Regression Networks (MVPNet), 3D UNet, and 3D UNet-based network for lesion detection, lesion segmentation, and lobe segmentation, respectively. Their algorithm analyzed the volume of abnormalities and the distance between lesion and pleura to diagnose the COVID-19, and it was concluded that the proposed algorithm outperformed three radiologists in terms of accuracy and sensitivity by achieving 94\% and $100 \%$, respectively. Table 3 summarizes the classification results for COVID-19/non-COVID-19 pneumonia cases.

3.4. COVID-19 Severity Classification Studies. Xiao et al. [61] implemented a pretrained network ResNet34 to diagnose COVID-19 severity. The experiments were performed using five-fold cross-validation, and 23,812 CT images of 408 patients were considered. They concluded that the model achieved the ROC AUC score of 0.987, and the prediction quality of detecting severity and nonseverity of $87.50 \%$ and $78.46 \%$.

Zhu et al. [62] proposed a model that was optimized by traditional CNN and VGG16 to stage the COVID-19 severity. A publicly available dataset was considered, and 113 COVID-19 confirmed cases were used to test their hypothesis. Obtained scores were compared by scores given by radiologists, and it was concluded that the top model achieved a correlation coefficient $\left(R^{2}\right)$ and mean absolute error of 0.90 and $8.5 \%$, respectively.

$\mathrm{Pu}$ et al. [63] proposed an approach that initially segmented lung boundary and major vessels at two $t$ points using UNet and registered these two images using a bidirectional elastic registration algorithm. Then, the average density of the middle of the lungs was used to compute a threshold to detect regions associated with pneumonia. Finally, the radiologist used to rate heat map accuracy in representing progression. In their study, two datasets that consisted of 192 CT scans were considered. Table 4 summarizes the key findings of the severity quantification studies. 


\begin{tabular}{|c|c|c|c|c|c|c|c|c|c|c|c|}
\hline 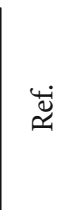 & 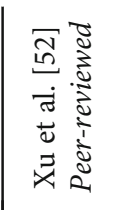 & 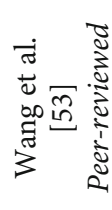 & 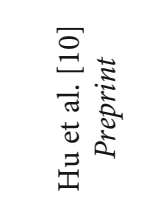 & 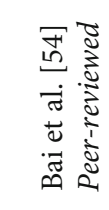 & 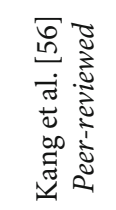 & 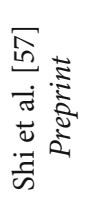 & 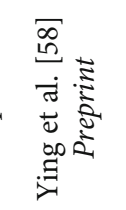 & 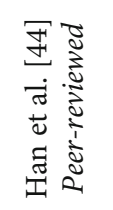 & 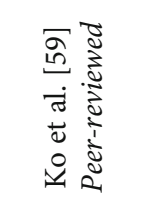 & 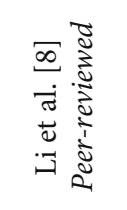 & 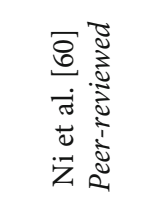 \\
\hline 立总 & $\underset{\infty}{\infty}$ & $\vec{\square}$ & 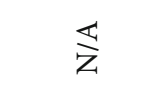 & $\overleftrightarrow{Z}$ & $\overleftrightarrow{Z}$ & 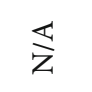 & $\infty$ & 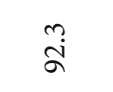 & $\overleftrightarrow{z}$ & $\overleftrightarrow{Z}$ & $\stackrel{\circ}{\stackrel{\circ}{\sigma}}$ \\
\hline 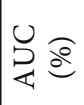 & 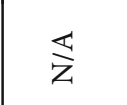 & $\begin{array}{l}8 \\
\stackrel{0}{0} \\
\infty\end{array}$ & $\begin{array}{l}\underset{\hat{~}}{\text { }} \\
\text {. }\end{array}$ & ณू & $\overleftrightarrow{z}$ & $\stackrel{\text { İ }}{\text { H̊ }}$ & nू & $\begin{array}{l}\infty \\
\infty \\
\infty\end{array}$ & $\stackrel{\circ}{\circ}$ & ஃ̊ & 艺 \\
\hline$\dot{y} \dot{x}$ & 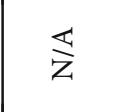 & $\begin{array}{l}8 \\
10 \\
10\end{array}$ & $\begin{array}{l}\text { 움 } \\
\text { Li } \\
\infty\end{array}$ & ஜ & $\stackrel{n}{\llcorner ૂ}$ & $\underset{\infty}{\stackrel{\infty}{\infty}}$ & $\infty$ & $\stackrel{m}{+}$ & $\begin{array}{l}\hat{\infty} \\
\stackrel{\alpha}{\alpha}\end{array}$ & $\overleftrightarrow{z}$ & హ゙ \\
\hline 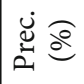 & $\frac{m}{\infty}$ & 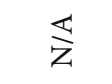 & 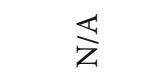 & $\overleftrightarrow{\overleftarrow{Z}}$ & 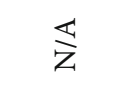 & $\overleftrightarrow{Z}$ & 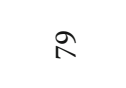 & ஙू & $\overleftrightarrow{z}$ & $\overleftrightarrow{z}$ & $\overleftrightarrow{Z}$ \\
\hline 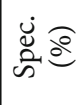 & $\frac{\varangle}{Z}$ & $\stackrel{\stackrel{m}{ }}{i}$ & $\begin{array}{l}\infty \\
\infty \\
\infty \\
\infty\end{array}$ & ฉ & సু & $\stackrel{\infty}{\infty}_{\infty}^{m}$ & 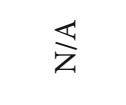 & $\overleftrightarrow{z}$ & $\stackrel{\circ}{\circ}$ & ๙ & $\stackrel{\mathscr{\sim}}{\sim}$ \\
\hline 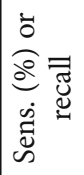 & $\hat{\infty}$ & $\begin{array}{l}\stackrel{n}{2} \\
\stackrel{n}{x}\end{array}$ & $\begin{array}{l}\vec{\lambda} \\
\infty \\
\infty\end{array}$ & $\kappa$ & 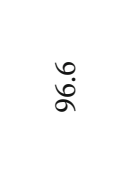 & $\hat{\circ}$ & ஜ & $\stackrel{\text { Ln }}{2}$ & $\begin{array}{l}\infty \\
12\end{array}$ & \& & 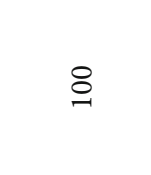 \\
\hline 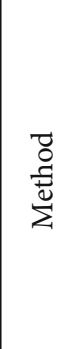 & 朰芯芯 & 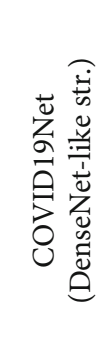 & 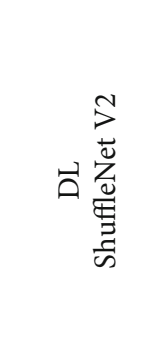 & 荒莒 & 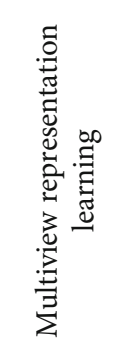 & 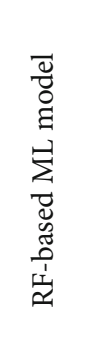 & 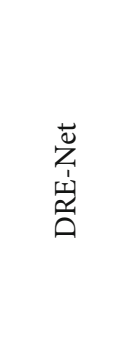 & $\begin{array}{l}\sum_{1}^{\mid} \\
\text {官 } \\
\text { 安 }\end{array}$ & 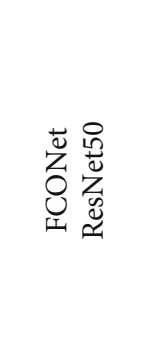 & 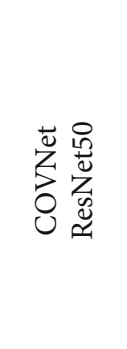 & 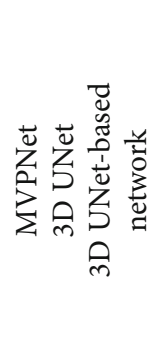 \\
\hline 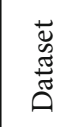 & 莺 & 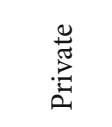 & 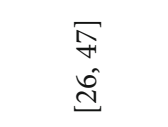 & 苛 & 泀 & 苛 & 泀 & $\stackrel{5}{:}$ & 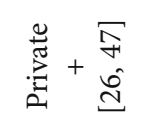 & 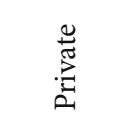 & 泀 \\
\hline $\begin{array}{l}\frac{n}{\tilde{u}} \\
\frac{\tilde{\omega}}{0} \\
\tilde{\omega}\end{array}$ & 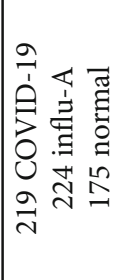 & 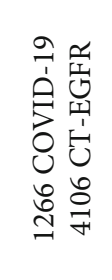 & 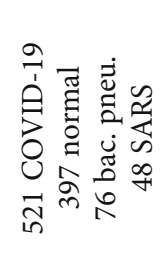 & 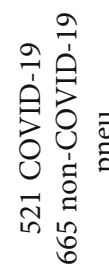 & 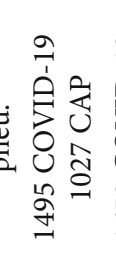 & 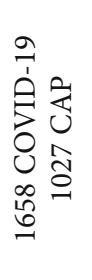 & 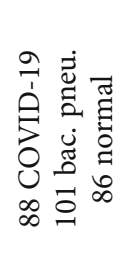 & 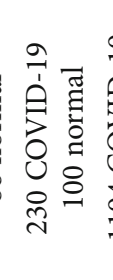 & 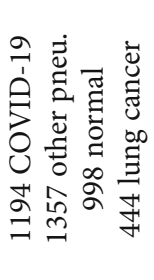 & 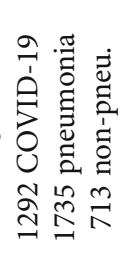 & 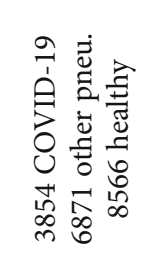 \\
\hline $\begin{array}{l}\dot{\infty} \\
\dot{\tilde{J}} \\
\dot{U}\end{array}$ & 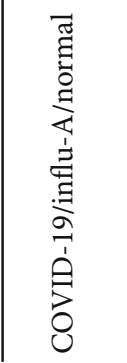 & 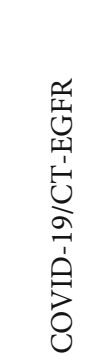 & 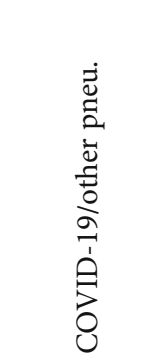 & 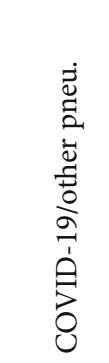 & $\frac{\text { 㐫 }}{2}$ & 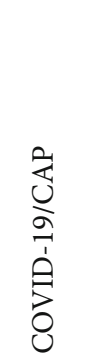 & 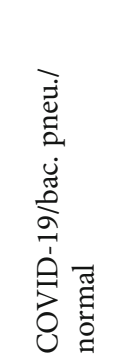 & 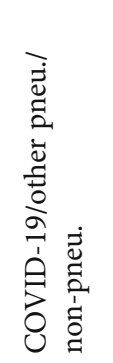 & 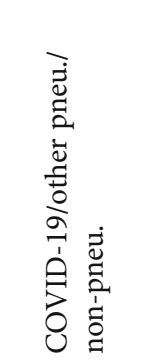 & 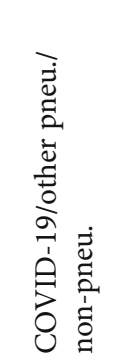 & 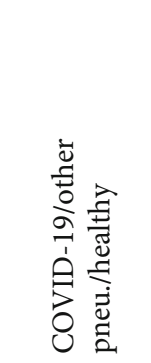 \\
\hline
\end{tabular}


TABLe 4: COVID-19 severity classification results. Class.: classification; Sens.: sensitivity; Spec.: specificity; Prec.: precision; AUC: area under the curve; Ref.: reference.

\begin{tabular}{|c|c|c|c|c|c|c|c|c|}
\hline Class. & Subjects & Dataset & Method & Sens. (\%) or recall & Spec. (\%) & Prec. (\%) & AUC (\%) & Ref. \\
\hline $\begin{array}{l}\text { COVID-19 severe/ } \\
\text { nonsevere }\end{array}$ & 23,812 COVID-19 & Private & ResNet34 & N/A & N/A & 81.3 & 98.7 & $\begin{array}{c}\text { Xiao et al. [61] } \\
\text { Peer-reviewed }\end{array}$ \\
\hline $\begin{array}{l}\text { COVID-19 severity } \\
\text { score }\end{array}$ & 131 COVID-19 & {$[21]$} & $\begin{array}{c}\text { CNN } \\
\text { VGG16 }\end{array}$ & $\mathrm{N} / \mathrm{A}$ & $\mathrm{N} / \mathrm{A}$ & NA & NA & $\begin{array}{l}\text { Zhu et al. [62] } \\
\text { Peer-reviewed }\end{array}$ \\
\hline $\begin{array}{l}\text { COVID-19 severity } \\
\text { and progression }\end{array}$ & $\begin{array}{l}72 \text { COVID-19 } \\
120 \text { others }\end{array}$ & Private & $\begin{array}{c}\text { UNet } \\
\text { BER Algorithm }\end{array}$ & 95 & 84 & N/A & $\mathrm{N} / \mathrm{A}$ & $\begin{array}{l}\text { Pu et al. [63] } \\
\text { Peer-reviewed }\end{array}$ \\
\hline
\end{tabular}

\section{Discussion}

The 13 of the 30 published articles considered in this review have been published as preprints, while the 17 of them have been published in journals after the peer-review process. Regardless of its form of publication, machine learning and deep learning have been the focus of these studies. In particular, deep learning approaches such as CNN, which performed the feature extraction process automatically, were widely used in these researches.

Besides, pretrained networks were commonly used for the segmentation, feature extraction, and classification stages. Especially DenseNet121, ResNet50, ShuffleNet V2 were successfully reported by the researchers in the classification stages, while successful results were obtained with the images produced by UNet ++ at the segmentation stage. It was pointed out by the researchers that many of the developed systems were modeled using the modifications or improvements pretrained networks to improve the classification accuracy of COVID-19 in CT images after preprocessing and segmentation stages. This has shown that widely used pretrained networks can be used very successfully at every stage of image classification. Some researchers classified COVID-19 cases using machine learning techniques instead of using deep learning approaches by extracting the features from the images and achieved high recognition results. This brings essential advantages in terms of learning speed.

However, while the images used are not standard and performing experiments on different image databases in each research does not make it possible to make a comprehensive comparison, it contributes to deduce general opinion. While the $k$-fold cross-validation is time-consuming, a few of the researches used it, and most of the researchers performed experiments using a hold-out method, which is based on dividing the dataset into training and testing set with defined percentages. However, this makes it challenging to analyze the consistency of the models, but it does not reduce the importance of performed experiments, obtained results, and the role of artificial intelligence in the fight against COVID-19.

\section{Conclusions}

COVID-19 continues to spread around the globe. New classification and prediction models using AI, together with more publicly available datasets, have been arising increasingly. However, the majority of the studies are from the preprint literature and have not peer-reviewed. Furthermore, many of them have different classification tasks. Some of the studies have been conducted with very limited data. The data used in the studies might have come from different institutions and different scanners. Therefore, preprocessing of the data to make the radiographic images more similar and uniform is important in terms of providing more efficient analysis and consistency. The lack of demographic and clinical information of the patients is another limitation of these studies. We believe as the more dataset on COVID-19 with are available, the more accurate studies will be conducted. These findings are promising for AI to be used in the clinic as a supportive system for physicians in the detection of COVID-19.

\section{Data Availability}

The data used to support the findings of this study are included within the article.

\section{Conflicts of Interest}

The authors declare that there is no conflict of interest regarding the publication of this paper.

\section{Authors' Contributions}

Ilker Ozsahin and Boran Sekeroglu contributed equally to this work.

\section{References}

[1] World Health OrganizationAugust 2020, https://www.who .int/emergencies/diseases/novel-coronavirus-2019.

[2] WorldometerAugust 2020, https://www.worldometers.info/ coronavirus/.

[3] F. Shi, J. Wang, J. Shi et al., "Review of artificial intelligence techniques in imaging data acquisition, segmentation and diagnosis for COVID-19," IEEE Reviews in Biomedical Engineering, 2020.

[4] H. Bai, B. Hsieh, Z. Xiong et al., "Performance of radiologists in differentiating COVID-19 from viral pneumonia on chest CT," Radiology, vol. 296, no. 2, pp. E46-E54, 2020.

[5] Y. Fang, H. Zhang, J. Xie et al., "Sensitivity of chest CT for COVID-19: comparison to RT-PCR," Radiology, vol. 296, no. 2, pp. E115-E117, 2020.

[6] S. S. Hare, A. N. Tavare, and V. Dattani, "Validation of the British Society of Thoracic Imaging guidelines for COVID-19 chest radiograph reporting," Clinical Radiology, vol. 75, no. 9, 2020. 
[7] F. Pesapane, M. Codari, and F. Sardanelli, "Artificial intelligence in medical imaging: threat or opportunity? Radiologists again at the forefront of innovation in medicine," European Radiology Experimental, vol. 2, no. 1, 2018.

[8] L. Li, L. Qin, Z. Xu et al., "Using Artificial intelligence to Detect COVID-19 and Community-acquired pneumonia Based on Pulmonary CT: Evaluation of the Diagnostic Accuracy," Radiology, vol. 296, no. 2, pp. E65-E71, 2020.

[9] M. Z. Alom, M. M. S. Rahman, M. S. Nasrin, T. M. Taha, and V. K. Asari, "COVID MTNet: COVID-19 detection with multi-task deep learning approaches," arXiv preprint arXiv, 2004, https://arxiv.org/abs/2004.03747.

[10] R. Hu, G. Ruan, S. Xiang, M. Huang, Q. Liang, and J. Li, “Automated Diagnosis of COVID-19 Using Deep Learning and Data Augmentation on Chest CT," medRxiv, 2020, https://medRxiv .org/abs/2020.04.24.20078998.

[11] N. Ma, X. Zhang, H. Zheng, and J. Sun, "ShuffleNet V2: practical guidelines for efficient CNN architecture design," Computer Vision - ECCV 2018: 15th European Conference, Munich, Germany, September 8-14, 2018, Proceedings, Part XIV, Springer International Publishing, vol. 11218, pp. 122138, 2018.

[12] O. Gozes, M. Frid-Adar, H. Greenspan et al., "Rapid AI development cycle for the coronavirus (COVID-19) pandemic: initial results for automated detection \& patient monitoring using deep learning CT image analysis," arXiv, 2020, https://arXiv:2003.05037.

[13] H. Kassani, P. H. K. Sara, M. J. Wesolowski, K. A. Schneider, and R. Deters, "Automatic detection of coronavirus disease (COVID-19) in X-ray and CT images: a machine learning based approach," arXiv, vol. 10641, 2004.

[14] M. Sandler, A. Howard, M. Zhu, A. Zhmoginov, and L.C. Chen, "MobileNetV2: inverted residuals and linear bottlenecks," in 2018 IEEE/CVF Conference on Computer Vision and Pattern Recognition, pp. 4510-4520, Salt Lake City, UT, USA, 2018.

[15] G. Huang, Z. Liu, L. Van Der Maaten, and K. Weinberger, "Densely connected convolutional networks," in Proceedings of The Ieee Conference On Computer Vision And Pattern Recognition, pp. 4700-4708, Honolulu, HI, USA, 2017.

[16] F. Chollet, "Xception: deep learning with depthwise separable convolutions," in 2017 IEEE Conference on Computer Vision and Pattern Recognition (CVPR), pp. 1800-1807, Honolulu, HI, USA, 2017.

[17] C. Szegedy, V. Vanhoucke, S. Ioffe, J. Shlens, and Z. Wojna, "Rethinking the inception architecture for computer vision," in 2016 IEEE Conference on Computer Vision and Pattern Recognition (CVPR), pp. 2818-2826, Las Vegas, NV, USA, 2016.

[18] C. Szegedy, S. Ioffe, V. Vanhoucke, and A. Alemi, "Inception$\mathrm{v} 4$, inception-ResNet and the impact of residual connections on learning, AAAI'17," in Proceedings of the Thirty-First AAAI Conference on Artificial Intelligence, pp. 4278-4284, San Francisco, CA, USA, 2017.

[19] K. He, X. Zhang, S. Ren, and J. Sun, "Deep residual learning for image recognition," in 2016 IEEE Conference on Computer Vision and Pattern Recognition (CVPR), pp. 770-778, Las Vegas, NV, 2016.

[20] A. Jaiswal, N. Gianchandani, D. Singh, V. Kumar, and M. Kaur, "Classification of the COVID-19 infected patients using DenseNet201 based deep transfer learning," Journal of Biomolecular Structure \& Dynamics, pp. 1-8, 2020.
[21] J. P. Cohen, P. Morrison, L. Dao, K. Roth, T. Q. Duong, and M. Ghassemi, "Covid-19 image data collection," arXiv, vol. 11988, 2006https://github.com/ieee8023/covid-chestxraydataset.

[22] Kaggle, "RSNA Pneumonia Detection Challenge," 2020, https://www.kaggle.com/c/rsna-pneumonia-detectionchallenge.

[23] Kaggle, "SARS-COV-2 CT-Scan Dataset," https://www.kaggle .com/plameneduardo/sarscov2-ctscan-dataset.

[24] K. Hun and R. Wen, "COVID19 Dataset," https://github.com/ KevinHuRunWen/COVID-19.

[25] https://github.com/UCSD-AI4H/COVID-CT.

[26] COVID-19 Database, "Italian Society of Medical and Interventional Radiology (SIRM)," https://www.sirm.org/en/category/ articles/covid-19-database/.

[27] ChainZhttp://www.ChainZ.cn.

[28] S. G. Armato, G. McLennan, L. Bidaut et al., "The lung image database consortium (LIDC) and image database resource initiative (IDRI): a completed reference database of lung nodules on CT scans," Medical physics, vol. 38, no. 2, pp. 915-931, 2011.

[29] Kaggle, "Chest X-Ray Images (Pneumonia) dataset," 2020, https://www. http://kaggle.com/paultimothymooney/chestxray-pneumonia.

[30] C. Jin, W. Chen, Y. Cao et al., "Development and evaluation of an AI system for COVID-19 diagnosis," MedRxiv, 2020, https://medRxiv.org/abs/2020.03.20.20039834.

[31] A. Depeursinge, A. Vargas, A. Platon, A. Geissbuhler, P. A. Poletti, and H. Müller, "Building a reference multimedia database for interstitial lung diseases," Computerized Medical Imaging and Graphics, vol. 36, no. 3, pp. 227-238, 2012.

[32] D. Singh, V. Kumar, M. K. Vaishali, and M. Kaur, "Classification of COVID-19 patients from chest CT images using multiobjective differential evolution-based convolutional neural networks," European Journal of Clinical Microbiology \& Infectious Diseases, vol. 39, no. 7, pp. 1379-1389, 2020.

[33] A. Amyar, R. Modzelewski, and S. Ruan, "Multi-task deep learning based ct imaging analysis for covid-19: classification and segmentation," medRxiv, 2020, https://medRxiv.org/abs/ 2020.04.16.20064709.

[34] S. Ahuja, B. K. Panigrahi, N. Dey, T. Gandhi, and V. Rajinikanth, "Deep transfer learning-based automated detection of COVID-19 from lung CT scan slices," Applied Intelligence, 2020.

[35] B. Liu, X. Gao, M. He, L. Liu, and G. Yin, "A fast online COVID-19 diagnostic system with chest CT scans," in Proceedings of KDD 2020, New York, NY, USA, 2020.

[36] M. Barstugan, U. Ozkaya, and S. Ozturk, "Coronavirus (COVID-19) classification using CT images by machine learning methods," ArXiv, 2020, https://arXiv:2003.09424.

[37] X. Wang, X. Deng, Q. Fu et al., "A weakly-supervised framework for COVID-19 classification and lesion localization from chest CT," IEEE transactions on medical imaging, vol. 39, no. 8, pp. 2615-2625, 2020.

[38] J. Chen, L. Wu, J. Zhang et al., “Deep learning-based model for detecting 2019 novel coronavirus pneumonia on highresolution computed tomography: a prospective study," medRxiv, 2020, https://medRxiv.org/abs/2020.02.25.20021568.

[39] S. Jin, B. Wang, H. Xu et al., "AI-assisted CT imaging analysis for COVID-19 screening: building and deploying a medical AI 
system in four weeks," medRxiv, 2020, https://medRxiv.org/ abs/2020.03.19.20039354.

[40] Y. Chen, J. Li, H. Xiao, X. Jin, S. Yan, and J. Feng, "Dual path networks," in 31st Conference on Neural Information Processing Systems (NIPS 2017), pp. 4467-4475, Long Beach, CA, USA, 2017.

[41] F. Wang, M. Jiang, C. Qian et al., "Residual attention network for image classification," in 2017 IEEE Conference on Computer Vision and Pattern Recognition (CVPR), pp. 31563164, Honolulu, HI, USA, 2017.

[42] Y. Pathak, P. K. Shukla, A. Tiwari, S. Stalin, S. Singh, and P. K. Shukla, "Deep transfer learning based classification model for COVID-19 disease," IRBM, 2020.

[43] M. Polsinelli, L. Cinque, and G. Placidi, "A light CNN for detecting COVID-19 from CT scans of the chest," arXiv preprint arXiv, vol. 12837, 2004.

[44] Z. Han, B. Wei, Y. Hong et al., "Accurate screening of COVID19 using attention based deep 3D multiple instance learning," IEEE Transactions on Medical Imaging, vol. 39, no. 8, pp. 2584-2594, 2020.

[45] S. A. Harmon, T. H. Sanford, S. Xu et al., “Artificial intelligence for the detection of COVID-19 pneumonia on chest CT using multinational datasets," Nature Communications, vol. 11, no. 1, p. 4080, 2020.

[46] X. Li, X. Zeng, B. Liu, and Y. Yu, "COVID-19 infection presenting with CT halo sign," Radiol Cardiothorac Imaging, vol. 2, no. 1, article e200026, 2020.

[47] J. Zhao, Y. Zhang, X. He, and P. Xie, "Covid-ct-dataset: a ct scan dataset about covid-19," arXiv preprint arXiv, vol. 13865, 2003.

[48] “COVID-19 CT segmentation dataset," http:// medicalsegmentation.com/covid19/.

[49] COVID-CT Datasethttps://github.com/UCSD-AI4H/ COVID-CT.

[50] X. He, X. Yang, S. Zhang et al., "Sample-efficient deep learning for covid-19 diagnosis based on ct scans," medRxiv, 2020, https://medRxiv.org/abs/2020.04.13.20063941.

[51] M. E. H. Chowdhury, T. Rahman, A. Khandakar et al., "Can AI help in screening viral and covid-19 pneumonia?arXiv preprint arXiv," vol. 13145, 2003.

[52] X. Xu, X. Jiang, C. Ma et al., "A deep learning system to screen novel coronavirus disease 2019 pneumonia," Engineering, 2020.

[53] S. Wang, Y. Zha, W. Li et al., "A fully automatic deep learning system for COVID-19 diagnostic and prognostic analysis," European Respiratory Journal, vol. 56, no. 2, article 2000775, 2020.

[54] H. X. Bai, R. Wang, Z. Xiong et al., "AI augmentation of radiologist performance in distinguishing COVID-19 from pneumonia of other etiology on chest CT," Radiology, vol. 296, no. 3, pp. E156-E165, 2020.

[55] M. Tan and Q. V. Le, "EfficientNet: rethinking model scaling for convolutional neural networks," arXiv ePrints, vol. 11946, 1905.

[56] H. Kang, L. Xia, F. Yan et al., "Diagnosis of coronavirus disease 2019 (covid-19) with structured latent multi-view representation learning," IEEE Transactions on Medical Imaging, vol. 39, no. 8, pp. 2606-2614, 2020.

[57] F. Shi, L. Xia, F. Shan et al., "Large-scale screening of COVID19 from community acquired pneumonia using infection sizeaware classification,” arXiv, 2003, https://arXiv:2003.09860.
[58] Y. Song, S. Zheng, L. Li et al., "Deep learning enables accurate diagnosis of novel coronavirus (COVID-19) with CT images," MedRxiv, 2020, https://medRxiv.org/abs/2020.02.23 .20026930 .

[59] H. Ko, H. Chung, W. S. Kang et al., "COVID-19 pneumonia diagnosis using a simple 2D deep learning framework with a single chest CT Image: Model Development and Validation," Journal of Medical Internet Research, vol. 22, no. 6, article e19569, 2020.

[60] Q. Ni, Z. Sun, L. Qi et al., “A deep learning approach to characterize 2019 coronavirus disease (COVID-19) pneumonia in chest CT images," European Radiology, pp. 1-11, 2020.

[61] L. Xiao, P. Li, F. Sun et al., "Development and Validation of a Deep Learning-Based Model Using Computed Tomography Imaging for Predicting Disease Severity of Coronavirus Disease 2019," Frontiers in Bioengineering and Biotechnology, vol. 8, 2020.

[62] J. Zhu, B. Shen, A. Abbasi, M. Hoshmand-Kochi, H. Li, and T. Q. Duong, "Deep transfer learning artificial intelligence accurately stages COVID-19 lung disease severity on portable chest radiographs," PLoS One, vol. 15, no. 7, article e0236621, 2020.

[63] J. Pu, J. K. Leader, A. Bandos et al., "Automated quantification of COVID-19 severity and progression using chest CT images," European Radiology, 2020. 